\title{
Comparative Genome Analysis of Livestock and Human Colistin-Resistant Escherichia coli Isolates from the Same Household
}

This article was published in the following Dove Press journal: Infection and Drug Resistance

\author{
Ryuji Kawahara' \\ Takahiro Yamaguchi' \\ Yoshimasa Yamamoto ${ }^{2,3}$ \\ 'Division of Microbiology, Osaka Institute \\ of Public Health, Osaka, Japan; ${ }^{2}$ Graduate \\ School of Pharmaceutical Sciences, Osaka \\ University, Suita, Japan; ${ }^{3}$ Life Science \\ Research Center, Gifu University, Gifu, \\ Japan
}

Background: Emergence and dissemination of colistin-resistant bacteria that harbor mobile colistin resistance $(\mathrm{mcr})$ genes pose a dire challenge for the treatment of intractable infections caused by multidrug-resistant bacteria. Current findings on colistin-resistant bacteria in both humans and livestock of the same households highlight the need to identify the dissemination mechanisms of colistin-resistant bacteria.

Methods: In this study, a comparative genome analysis of colistin-resistant Escherichia coli isolates from livestock and humans of the same household was performed to clarify the possible dissemination mechanism of $\mathrm{mcr}$ genes among bacteria. Pulsed-field gel electrophoresis and whole-genome sequencing followed by sequence typing of the isolates were performed for assessment of the samples.

Results: The study revealed that two colistin-resistant $E$. coli isolates, one each from a pig and a chicken, were phylogenetically similar but not identical to the human isolates obtained from the same household. The comparative genome analysis revealed that the chicken isolate and a human isolate shared the same IncHl2 plasmid harboring the $m c r$ transposon ( $m c r-1$-PAP2). The pig isolate and the other human isolate retained the $m c r-1$ transposon on the chromosome, with the pig isolate carrying the complete $m c r$ transposon (ISApl1-mcr-1-PAP2-ISApl1) and the human isolate carrying the incomplete $m c r$ transposon (ISApl1-mcr-1-PAP2).

Conclusion: The results of the study confirm the distribution of colistin-resistant bacteria and subsequent transmission of the resistance gene-carrying transposon between livestock and humans of the same household. To the best of our knowledge, this is the first report on genomic analysis of colistin-resistant $E$. coli isolates obtained from livestock and residents of the same household.

Keywords: colistin-resistance, multidrug resistance, comparative genome analysis, wholegenome sequencing, $\mathrm{mcr}$

\section{Introduction}

Colistin, one of the few treatment options for intractable infectious diseases caused by multidrug-resistant Gram-negative bacteria, ${ }^{1}$ is a popular drug in livestock breeding; it is not only used to treat infections but also as a livestock growth promoter and infection protective agent. ${ }^{2}$ The overuse and misuse of colistin is considered responsible for the emergence and dissemination of colistin-resistant (COR) bacteria in animal husbandry. In addition, since colistin resistance can be transferred by a mobile colistin resistance gene, $m c r$, wide dissemination of COR bacteria can be expected in livestock as well as humans. In fact, livestock is considered a reservoir for COR bacteria. ${ }^{3}$
Correspondence: Yoshimasa Yamamoto Life Science Research Center, Gifu University, I-I Yanagido, Gifu, 50I-I I94, Japan

Tel/Fax +8I-58-230-6239

Email yyamamot@gifu-u.ac.jp
Infection and Drug Resistance 2021:|4 84|-847 
Our recent studies have reported wide dissemination of COR bacteria in human residents of communities where livestock carried COR bacteria. ${ }^{4-6}$ The results of these studies suggest that horizontal transmission of resistance may occur between livestock and human residents in the same community, including transmission of specific resistant isolates. However, there is a lack of direct evidence supporting the transmission of COR bacteria and/or the resistance gene, $m c r$, in the community.

A recent study on $m c r-1$-harboring plasmids obtained from COR Escherichia coli isolates from humans and livestock has reported the details of $m c r$-1-carrying IncI2 plasmid, ${ }^{7}$ which is one of the major replicons for the $\mathrm{mcr}$ 1-carrying plasmids. $^{8}$ However, these studies analyzed clinical isolates from patients and livestock isolates retrospectively. In this study, we aimed to study the transmission of $m c r-1$ between COR E. coli isolates from humans and domestic animals in the same household by performing a comparative genomic analysis of these isolates.

\section{Materials and Methods}

\section{Bacterial Strains}

COR E. coli isolates, strains 2018-18.25PC and 2018-18.25CC, were previously isolated from rectal swab specimens of pig and chicken, respectively, in the backyard of a household in a rural community in Vietnam. ${ }^{4}$ Human COR E. coli isolates, 2017.02.01CC, 2017.02.02CC, and 2018-02.2CC, were also previously isolated from the fecal specimens of two family members of the same household as the livestock. ${ }^{5}$ The study was approved by the Ethics Committees of Osaka University (Osaka, Japan) and Thai Binh University of Medicine and Pharmacy (Thai Binh, Vietnam). The characteristics of these isolates are summarized in Table 1.

\section{Pulsed-Field Gel Electrophoresis (PFGE)}

Xbal-digested genomic DNA samples from the COR E. coli isolates were analyzed on a CHEF-DR III System (Bio-Rad, Hercules, CA, USA) following the methods reported previously. ${ }^{9}$

\section{Genome Sequencing}

In this study, we analyzed the strains $2018-18.25 \mathrm{PC}$ and 2018-18.25CC obtained from a pig and a chicken, respectively, by genome sequencing. Whole-genome sequencing and assembly of the isolates as well as of the plasmid harboring $m c r-1$, were performed using the Illumina

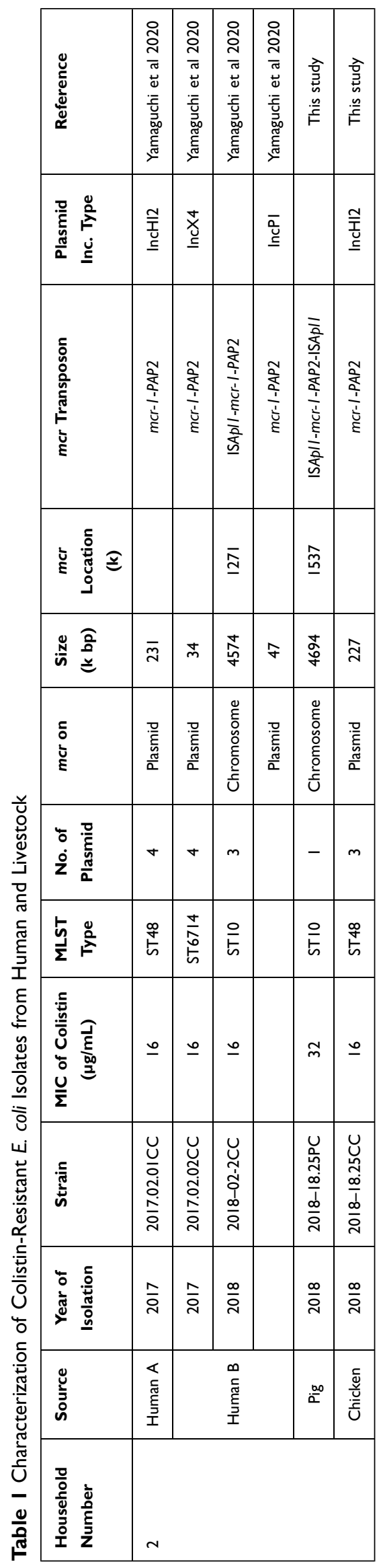



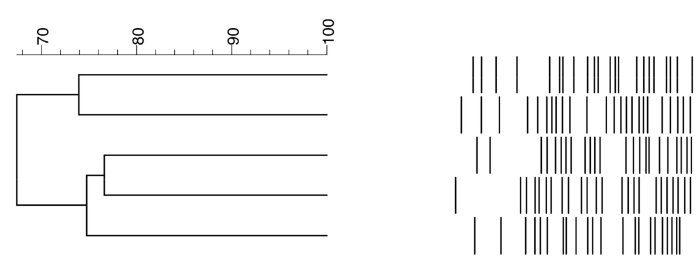

$\begin{array}{lll}\text { Strain } & \text { Source } & \underline{\text { MLST }} \\ \text { 2017.02.02CC } & \text { Human } & \text { ST6714 } \\ \text { 2018-18.25PC } & \text { Pig } & \text { ST10 } \\ \text { 2017.02.01CC } & \text { Human } & \text { ST48 } \\ \text { 2018-18.25CC } & \text { Chicken } & \text { ST48 } \\ \text { 2018-02-2CC } & \text { Human } & \text { ST10 }\end{array}$

Figure I Dendrogram of pulsed-field gel patterns of isolates from human and livestock.

MiSeq (Illumina Inc., CA, USA) and MinION (Oxford Nanopore Technologies, London, UK) sequencers, as described previously. ${ }^{10}$ The genome sequences were automatically annotated using the DDBJ Fast Annotation and Submission Tool Pipeline (https://dfast.nig.ac.jp). Genome analysis was performed using the Geneious R11 software (Biomatters, Ltd., NZ). Antibiotic resistance genes and replicon typing were conducted in ResFinder $4.0^{11}$ and PlasmidFinder 1.3 on the Center for Genomic Epidemiology website (http://www.genomicepidemiology. org). Multilocus sequence type (MLST) analysis was performed using a MLST software (https://github.com/tsee mann/mlst). The mcr-1-bearing IncHI2 plasmids were compared with the reference plasmid pECJS-59-244 ${ }^{11}$ using BRIG ${ }^{12}$ and visualized with Easyfig. ${ }^{13}$

Genome analysis of the human isolates was performed previously, and the analyzed data were used in this study. ${ }^{14}$

\section{Results}

\section{Comparison of Livestock- and Human-Derived Strains Isolated from the Same Household}

The phylogenetic relation, $m c r-1$ location, and $m c r$ transposon structure were assessed for five isolates. Two isolates were obtained from a chicken and a pig each, which were maintained in the backyard of the household; one isolate was obtained from a human member of the same household at the time of the isolation of the livestock strains, and two isolates were obtained from the human members of the household 10 months prior to the isolation of the livestock strains. The phylogenetic likelihood of these five isolates was assessed using PFGE, and the results are shown in Figure 1. None of the five isolates belonged to the same clone. However, the pig isolate 2018-18.25PC and the human isolate 2018-02.2CC belonged to sequence type (ST) 10, and the chicken isolate 2018-18.25CC and the human isolate 2017.02.01CC to ST48.
Comparative genomic analysis was performed to assess the genetic details of $m c r-1$ in these isolates. Similar to the human isolates, the livestock isolates carried $m c r-1$ on either the chromosome or a plasmid (Table 1). The chicken isolate 2018-18.25CC harbored $m c r-1$ on plasmid IncHI2 as observed in the human isolate 2017.02.01CC. Although both isolates belonged to ST48, phylogenetically, they were different. The pig isolate 2018-18.25PC belonged to ST10 and harbored $m c r-1$ on the chromosome similar to the human isolate 2018-02.2CC; however, these two isolates were different (Figure 1). The human isolate 2017.02.02CC was also different from the livestock isolates.

\section{Comparative Details of $m c r-I$ in Human and Livestock Isolates}

The results of the comparative analysis of the $\mathrm{mcr}$ gene showed that both the human strain 2018-02-2CC and the pig strain 2018-18.25PC retained the $m c r-1$ transposon on the chromosome; however, the human strain had an incomplete transposon, ISApl1-mcr-1-PAP2, whereas the pig strain possessed the complete transposon, ISApl1- $m c r$ -1-PAP2-ISApl1 (Table 1). Furthermore, the location of mcr-1 on both chromosomes was genetically related despite the strains being different.

Sequence of $m c r-1$-plasmid IncHI2 found in the human strain 2017.02.01CC and the chicken strain 2018-18.25CC showed that both plasmids (p2017.02.01CC, and p2018$18.25 \mathrm{CC}$ ) were similar (query cover; 88\%, identity; 99.99\%, Figure 2) except for some drug resistance genes in the multidrug resistance (MDR) region of $\mathrm{p} 2018$ 18.25CC, which were inserted in the reverse direction (Figure 3). IncHI2 plasmid carries a large MDR region containing antibiotic resistance genes other than $m c r-1 .{ }^{15}$ Therefore, a comparative study with the plasmids from the isolated strains and the reference plasmid pECJS-59-244 (KX084394) was conducted. As shown in Figure 3, plasmids from the human and chicken isolates were similar in terms of plasmid organization to the reference plasmid. 


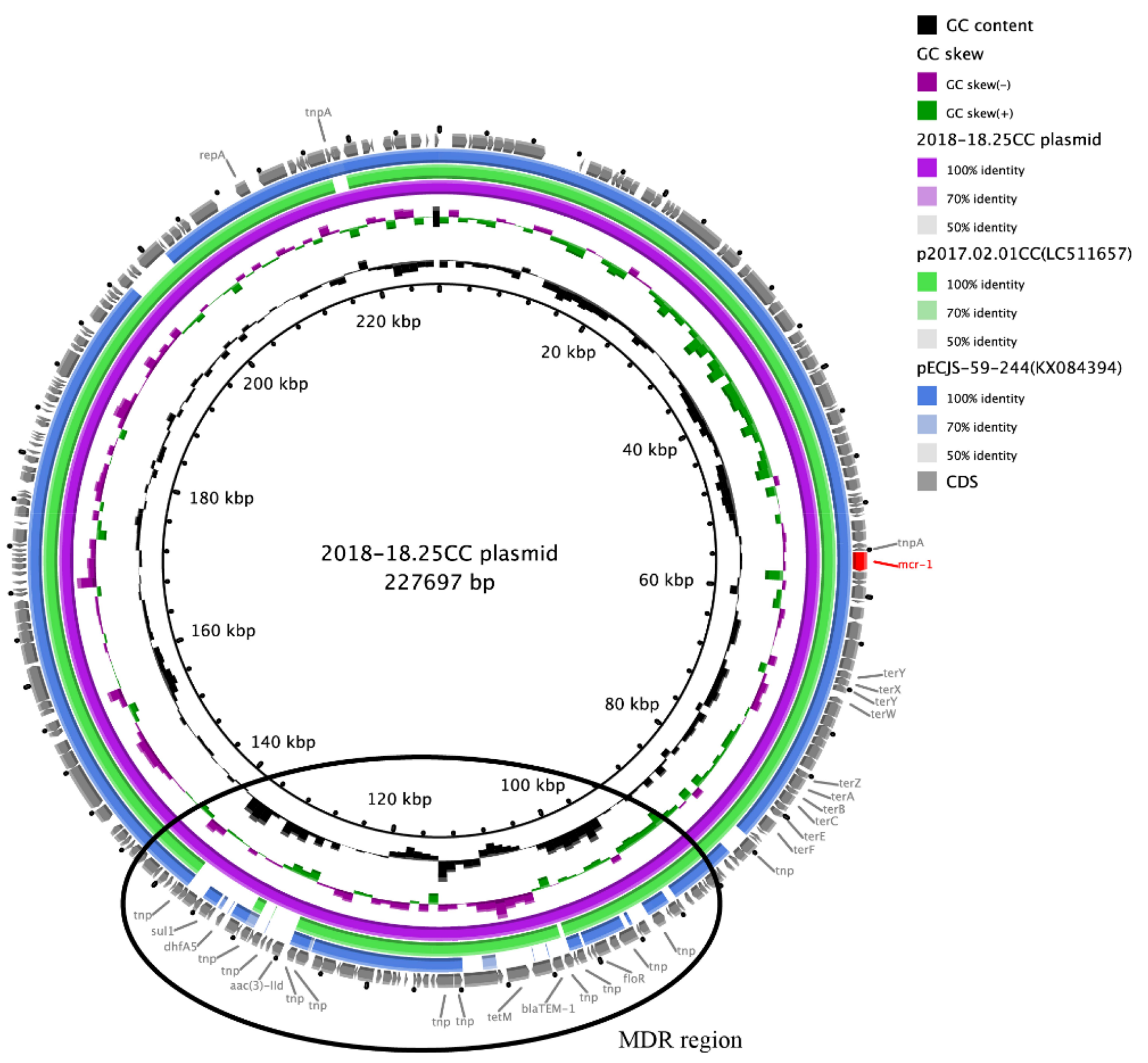

Figure 2 Sequence alignment of IncHI2 plasmids with mcr-I in isolates obtained from a human and a chicken of the same household. Plasmids $2018-18.25 C C$ and p2017.02.0ICC were present in the chicken and human isolates, respectively. The reference IncHI2 plasmid pECJS-59-244 was isolated from a pig in a previous study. The circle indicates the putative MDR region.

However, the MDR region of the human and chicken isolates lacked certain resistance genes present in the reference plasmid. Interestingly, IncHI2 isolated from the human and chicken isolates harbored $f l o R$, a gene coding for resistance against the veterinary drug florfenicol; this was similar to the reference IncHI2 (KX084394).

\section{Discussion}

The results of this study showed that human members of the household harbored COR E. coli strains with the same $\mathrm{ST}$ as that of the livestock. In particular, the COR E. coli ST48 strains were isolated from chicken as well as humans. These isolates were genetically similar but were different clones, as assessed by PFGE analysis. This indicates that these isolates were derived from the same clone and evolved by long-term adaptation to the hosts (humans and livestock) as well as environmental conditions. Furthermore, since this study was not conducted during a short-term epidemic of COR bacteria spreading in the community, it was highly unlikely to observe the same clone in both humans and livestock. Therefore, a clonal distribution of COR strains among livestock and humans was anticipated. In addition, identification of the same IncHI2 plasmid carrying $m c r-1$ in these isolates might 


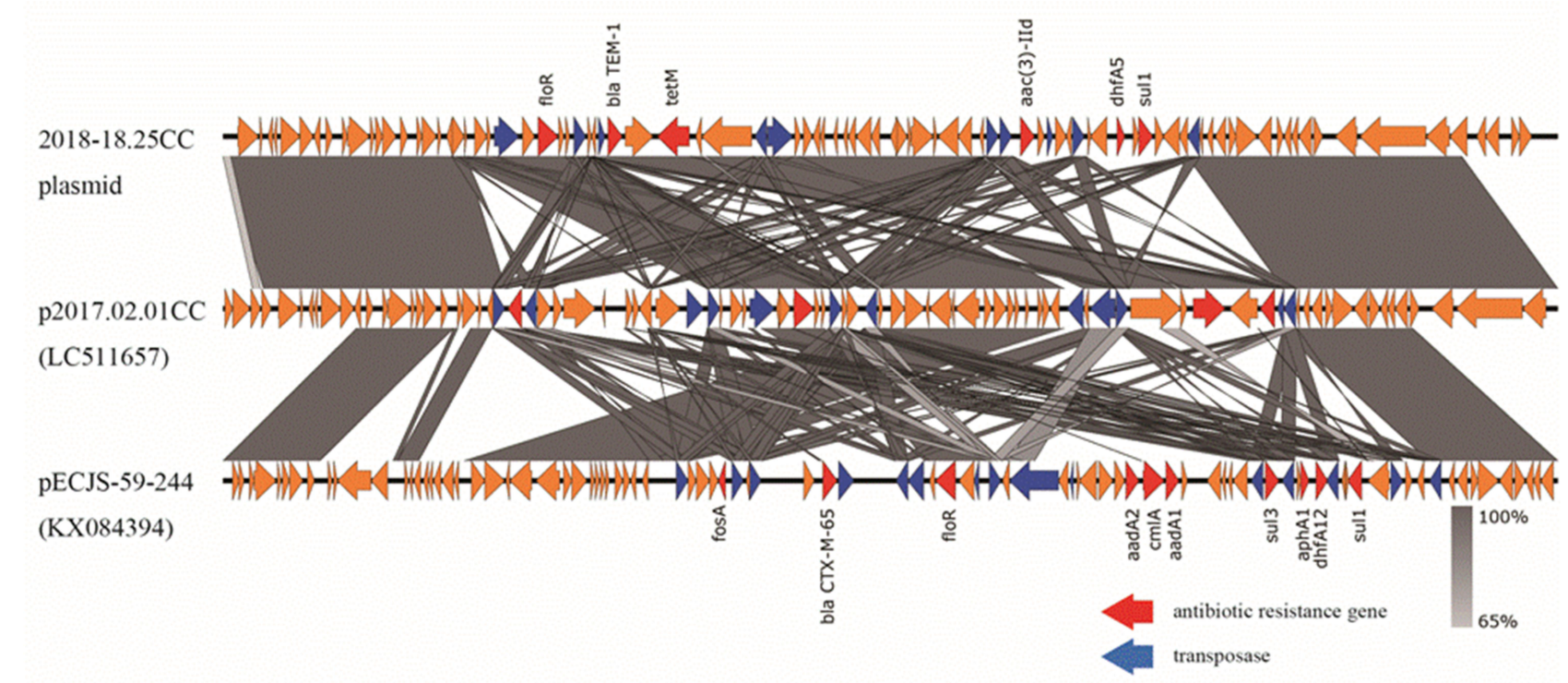

Figure 3 Sequence alignment of the putative MDR region in plasmids. Plasmids $2018-18.25 \mathrm{CC}$ and $\mathrm{p} 2017.02 .01 \mathrm{CC}$ were present in the chicken and human isolates, respectively. The reference IncHI2 plasmid pECJS-59-244 was isolated from a pig in a previous study.

provide the initial evidence for COR bacteria dissemination and subsequent spread of plasmids in the household.

The phylogenetic similarity of human and pig ST10 COR isolates was low. The COR E. coli ST10 isolate from the pig did not possess an mcr-1-carrying plasmid but carried a $m c r-1$ on the chromosome akin to the human ST10 isolate. However, unlike the human ST10 isolate, the structure of the mcr-1 transposon found in the pig isolate was well conserved. Since the insertion sequences (ISs) of the mcr-1 transposon TN6330 (ISApl1-mcr-1-PAP2-IS Apll) have dropped out during stabilization of the genes across generations, ${ }^{16,17}$ it is likely that transmission of TN6330 between plasmids, chromosomes, and strains may have occurred in the household over a long period. Therefore, the ST10 clone may have spread between humans and livestock like the ST48 clone. Furthermore, the presence of $f l o R$ on plasmids of both livestock and human isolates indicates the direction of this transmission. The occurrence of $f l o R$ is commonly associated with $\mathrm{mcr}-1$ and suggests animal origin of the isolate. ${ }^{8,17}$ However, more isolates need to be assessed to confirm this observation.

Since the discovery of $\mathrm{mcr}$ on plasmids, many studies have been conducted on mcr-carrying plasmids of COR bacteria isolated from various sources. ${ }^{7,8,18,19}$ Outcomes of a metadata analysis revealed that the mcr-carrying plasmids are diverse. ${ }^{19}$ Among these $m c r$-carrying plasmids, IncI2, IncX4, and IncHI2 plasmids are found most frequently, even in livestock isolates. The outcomes of the present study regarding $m c r-1$-harboring plasmid IncHI2 of the livestock COR isolate are consistent with those of previous studies. ${ }^{15}$

The IncHI2 plasmid is also known to actively acquire other foreign resistance genes. ${ }^{15}$ BLASTN and ResFinder analysis on the resistance gene database showed that the plasmids of the chicken isolate 2018-18.25CC and the human isolate 2017.02.01CC comprised MDR determinants, including floR, bla $a_{\mathrm{TEM}}$, tetM, aaC3, dfrA5, and sull. However, genes such as bla $_{\mathrm{CTX- \textrm {M }}}, f o s A$, and aad $A$, reported in the reference pig isolate obtained from farms in China, were not present in our isolates. ${ }^{15}$ Addition of MDR genes to $m c r$-carrying plasmids and the subsequent spread of the plasmid in the microbiomes of humans and livestock poses a threat to public health worldwide. The results of the present study showed that accumulation of resistance genes was limited in the isolates. However, accumulation of resistance genes can be expected in the future. Therefore, monitoring of MDR genes besides $\mathrm{mcr}$ in the plasmids of human and livestock microbiomes is urgently required.

The chromosomally encoded $m c r-1$ is important due to its stability. ${ }^{13,20}$ The presence of chromosomal $\mathrm{mcr}-1$ has been reported, but its frequency was low $(3.5 \%) .{ }^{20}$ In this study, although only two isolates from livestock were assessed genetically, the finding of chromosomal $\mathrm{mcr}-1$ in both of them is consistent with a previous study 
reporting the occurrence of chromosomal $\mathrm{mcr}-1$ in $36.8 \%$ of the isolates from healthy residents living in the same area. ${ }^{13}$ Therefore, the monitoring of chromosomal $\mathrm{mcr}$ in COR bacteria it is also important.

\section{Conclusion}

To the best of our knowledge, this is the first report of a direct comparative analysis of COR E. coli isolates from livestock and humans of the same household. The initial findings of this study suggest distribution of COR E. coli and subsequent transmission of the colistin resistance gene $m c r-1$ transposon between livestock and humans of the same household. The findings also support that dissemination of COR bacteria in communities occurs through domestic livestock. The study warrants further genetic analysis of COR bacteria isolates in communities where COR bacteria are disseminated.

\section{Abbreviations}

COR, colistin-resistant; PFGE, pulsed-field gel electrophoresis; MDR, multidrug resistance; IS, insertion sequence; MLST, multilocus sequence type; ST, sequence type.

\section{Data Sharing Statement}

The draft genome sequences of the COR E. coli strains 2018-18.25PC and sequences of the plasmid carrying $\mathrm{mcr}$ 1 of strain 2018-18.25CC were deposited in DDBJ/ GenBank under the accession numbers AP023286 and LC567843, respectively.

\section{Ethics Approval and Informed Consent}

The study was approved by the Ethics Committees of Osaka University (Osaka, Japan) and Thai Binh University of Medicine and Pharmacy (Thai Binh, Vietnam).

\section{Author Contributions}

All authors made a significant contribution to the work reported, whether that is in the conception, study design, execution, acquisition of data, analysis and interpretation, or in all these areas; took part in drafting, revising or critically reviewing the article; gave final approval of the version to be published; have agreed on the journal to which the article has been submitted; and agree to be accountable for all aspects of the work.

\section{Funding}

This work was supported by the Japan Society for the Promotion of Science KAKENHI (grants 17H01687 and 20H00561).

\section{Disclosure}

The authors report no conflicts of interest in this work.

\section{References}

1. Giamarellou H, Poulakou G. Multidrug-resistant gram-negative infections: what are the treatment options? Drugs. 2009;69(14):18 79-1901.

2. Rhouma M, Beaudry F, Letellier A. Resistance to colistin: what is the fate for this antibiotic in pig production? Int $J$ Antimicrob Agents. 2016;48(2):119-126.

3. Giamarellou H. Epidemiology of infections caused by polymyxin-resistant pathogens. Int J Antimicrob Agents. 2016;48 (6):614-621.

4. Kawahara R, Fujiya Y, Yamaguchi T, et al. Most domestic livestock possess colistin-resistant commensal Escherichia coli harboring $\mathrm{mcr}$ in a rural community in Vietnam. Antimicrob Agents Chemother. 2019;63(6):e00594-19.

5. Yamamoto Y, Kawahara R, Fujiya Y, et al. Wide dissemination of colistin-resistant Escherichia coli with the mobile resistance gene mcr in healthy residents in Vietnam. $J$ Antimicrob Chemother. 2019;74(2):523-524.

6. Yamamoto Y, Calvopina M, Izurieta R, et al. Colistin-resistant Escherichia coli with $m c r$ genes in the livestock of rural small-scale farms in Ecuador. BMC Res Notes. 2019;12:121.

7. Yoon EJ, Hong JS, Yang JW, et al. Detection of $m c r-1$ plasmids in Enterobacteriaceae isolates from human specimens: comparison with those in Escherichia coli isolates from livestock in Korea. Ann Lab Med. 2018;38(6):555-562.

8. Matamoros S, van Hattem JM, Arcilla MS, et al. Global phylogenetic analysis of Escherichia coli and plasmids carrying the $\mathrm{mcr}-1$ gene indicates bacterial diversity but plasmid restriction. Sci Rep. 2017;7:15364

9. Ribot EM, Fair MA, Gautom R, et al. Standardization of pulsed-field gel electrophoresis protocols for the subtyping of Escherichia coli O157:H7, Salmonella, and Shigella for PulseNet. Foodborne Pathog Dis. 2006;3(1):59-67.

10. Matsumoto Y, Kinjo T, Motooka D, et al. Comprehensive subspecies identification of 175 nontuberculous mycobacteria species based on 7547 genomic profiles. Emerg Microbes Infect. 2019;8(1):10 43-1053.

11. Bortolaia V, Kaas RS, Ruppe E, et al. ResFinder 4.0 for predictions of phenotypes from genotypes. J Antimicrob Chemother. 2020;75:3491.

12. Alikhan NF, Petty NK, Ben Zakour NL, et al. BLAST Ring Image Generator (BRIG): simple prokaryote genome comparisons. BMC Genomics. 2011;12:402.

13. Sullivan MJ, Petty NK, Beatson SA. Easyfig: a genome comparison visualizer. Bioinformatics. 2011;27(7):1009-1010.

14. Yamaguchi T, Kawahara R, Hamamoto K, et al. High prevalence of colistin-resistant Escherichia coli with chromosomally carried $m c r-1$ in healthy residents in Vietnam. mSphere. 2020;5(2):e00117-20.

15. Li R, Xie M, Zhang J, et al. Genetic characterization of mcr-1-bearing plasmids to depict molecular mechanisms underlying dissemination of the colistin resistance determinant. J Antimicrob Chemother. 2017;72(2):393-401.

16. Snesrud E, McGann P, Chandler M. The birth and demise of the IS Apl1- mcr-1-IS Apl1 composite transposon: the vehicle for transferable colistin resistance. mBio. 2018;9(1):e02381-17. 
17. Wang R, Dorp L, Shaw LP, et al. The global distribution and spread of the mobilized colistin resistance gene mor-1. Nat Commun. 2018;9(1):1179.

18. Ye H, Li Y, Li Z, et al. Diversified $m c r$-l-harbouring plasmid reservoirs confer resistance to colistin in human gut microbiota. mBio. 2016;7(2): e00177-16.

19. Lin $\mathrm{Y}$, Dong $\mathrm{X}, \mathrm{Wu} \mathrm{J}$, et al. Metadata analysis of $m c r-1$-bearing plasmids inspired by the sequencing evidence for horizontal transfer of antibiotic resistance genes between polluted river and wild birds. Front Microbiol. 2020;11:352.
20. Shen C, Zhong LL, Ma F, et al. Genomic patterns and characterizations of chromosomally-encoded $\mathrm{mcr}-1$ in Escherichia coli populations. Gut Pathog. 2020;12:55.

\section{Publish your work in this journal}

Infection and Drug Resistance is an international, peer-reviewed openaccess journal that focuses on the optimal treatment of infection (bacterial, fungal and viral) and the development and institution of preventive strategies to minimize the development and spread of resistance. The journal is specifically concerned with the epidemiology of

Submit your manuscript here: https://www.dovepress.com/infection-and-drug-resistance-journa antibiotic resistance and the mechanisms of resistance development and diffusion in both hospitals and the community. The manuscript management system is completely online and includes a very quick and fair peerreview system, which is all easy to use. Visit http://www.dovepress.com/ testimonials.php to read real quotes from published authors. 